\section{IMMUNOTHERAPY}

\section{$\mathrm{ICI}$ rechallenge in $\mathrm{mRCC}$}

Immune-checkpoint inhibitors (ICIs) targeting PD-1, PD-L1 or CTLA4 are components of various FDA-approved treatment regimens for metastatic renal cell carcinoma (mRCC). Despite the promising efficacy of these regimens, disease progression is inevitable for most patients. Now, data from two retrospective studies shed light on the safety and clinical activity of ICI rechallenge in this setting.

Gul et al. focused specifically on salvage therapy with nivolumab plus ipilimumab (nivo + ipi) in 45 patients previously treated with PD-1 or PD-L1 inhibitors, predominantly as monotherapy (in 60\%) or in combination with a VEGF receptor tyrosine kinase inhibitor (VEGFR-TKI; 18\%). The objective response rate (ORR) with salvage nivo + ipi was $20 \%$, all partial responses (PRs), with stable disease $(\mathrm{SD})$ in a further $16 \%$ of patients. Responses were observed regardless of whether patients had an objective response, $\mathrm{SD}$ or progressive disease
(PD) with prior ICI therapy (ORR $17 \%, 25 \%$ and $22 \%$, respectively). The median duration of response was 7 months, and the median progressionfree survival duration was 4 months. Interestingly, only 1 of 11 patients who had previously received a VEFGR-TKI, either with an ICI or alone in the most recent line of therapy, had a PR with nivo + ipi (ORR 9\%).

Ravi et al. evaluated the outcomes of 69 patients, most of whom had previously received an ICI plus a VEGF pathway-targeted agent (42\%), ICI monotherapy (39\%) or dual ICI therapy (13\%). ICI rechallenge was not restricted to any particular agent or combination, and most patients received single-agent ICI (38\%) or dual ICI (32\%) salvage therapy. The overall ORR was $23 \%$, and the rate of SD was $41 \%$. ORRs were similar in patients who received ICI monotherapy (30\%), dual ICI therapy (25\%) or an ICI plus a targeted agent (23\%), as well as in those who had received prior dual ICI (33\%) or VEGF pathway-targeted (17\%)

therapy. The ORR was $29 \%$ among patients with a response to prior ICI therapy (versus $15 \%$ and $21 \%$ in those with prior SD and $\mathrm{PD}$, respectively) and was $38 \%$ in those who discontinued prior ICI therapy owing to toxicities.

Grade 3-4 immune-related

The observation of responses regardless of treatment type and sequence emphasizes the need for predictive biomarkers gु

\title{
Myocardial infarction promotes breast cancer recurrence
}

Breast cancer survivors have an increased risk of cardiovascular events (myocardial infarction (MI) or stroke) owing to cancer-related factors including treatment-derived toxicities and lifestyle changes. Now, new data show that an MI promotes breast cancer progression in mouse models and increases the recurrence risk in humans.

$\mathrm{Ml}$ induced surgically 3 days after implantation of mouse breast cancer cells in the mammary fat pad was associated with a doubling of the tumour volume compared with that observed with sham surgery. Further analyses revealed greater numbers of proliferating cancer cells, monocytic myeloid-derived suppressor cells (mMDSCs) and regulatory T cells in mice with $\mathrm{MI}$; no differences were observed in other immune cell populations. "When we depleted

mMDSCs, the tumour growth advantage was abrogated in mice with an MI, and

These findings highlight the importance of characterizing the mechanisms

by which comorbidities can affect cancer progression

5
the number of antitumour $\mathrm{CD}^{+} \mathrm{T}$ cells increased," explains lead investigator Kathryn Moore.

Epigenetic alterations in myelopoietic progenitors underlie innate immune memory. To investigate a potential role for this mechanism in their model, the investigators performed ATAC-seq in bone marrow tissue. These analyses revealed increased chromatin accessibility in pathways related to stress responses and reduced accessibility in pathways regulating immune and inflammatory responses in mice with an MI versus control mice. Moreover, "we found that breast tumours grew faster in mice transplanted with bone marrow from mice with an Ml versus control mice, even though the recipient mice had never had an MI," describes Moore.
To assess the clinical relevance of these findings, two prospective cohort studies were retrospectively analysed. These studies involved 1,724 patients with early stage breast cancer and a median follow-up duration from diagnosis of 11.7 years. Compared with no cardiovascular event, a post-diagnosis cardiovascular event was associated with an adjusted increased risk of recurrence of $59 \%(95 \% \mathrm{Cl}$ 1.23-2.06) and of breast cancer-specific mortality of $60 \%$ (95\% Cl 1.16-2.22).

These findings highlight the importance of characterizing the mechanisms by which comorbidities can affect cancer progression. "Breast cancer survivors and their health-care providers should closely monitor risk factors and early symptoms of cardiovascular disease to reduce the risk of having an MI, as doing so might not only improve cardiovascular-related outcomes but also reduce cancer-specific mortality," Moore concludes.

Diana Romero

ORIGINAL ARTICLE Koelwyn, G. J. et al.

Myocardial infarction accelerates breast cancer via innate immune reprogramming. Nat. Med. https:// doi.org/10.1038/s41591-020-0964-7 (2020) 